RESEARCH ARTICLE

\title{
Multilevel Characterization of Antibody-Ligand Conjugates by CESI-MS
}

\author{
Bryan Fonslow ${ }^{\mathrm{a}}$, Gabor Jarvas ${ }^{\mathrm{b}, \mathrm{c},{ }^{*},}$ Marton Szigeti ${ }^{\mathrm{b}, \mathrm{c}}$ and Andras Guttman ${ }^{\mathrm{c}, \mathrm{d}}$
}

${ }^{a}$ The Scripps Research Institute, La Jolla, CA; ${ }^{b}$ Translational Glycomics Group, Research Institute of Biomolecular and Chemical Engineering, University of Pannonia, Veszprem, Hungary; ${ }^{c}$ Horváth Csaba Memorial Laboratory of Bioseparation Sciences, Research Centre for Molecular Medicine, Doctoral School of Molecular Medicine, Faculty of Medicine, University of Debrecen, Debrecen, Hungary, ${ }^{d}$ Sciex, Brea, CA

ARTICLE HISTORY

Received: February 27, 2019

Revised: March 19, 2020

Accepted: March 25, 2020

DOI:

$10.2174 / 1566524020666200415095830$

\begin{abstract}
Aim: To demonstrate the capabilities of our new capillary electrophoresis mass spectrometry method, which facilitates highly accurate relative quantitation of modification site occupancy of antibody-ligand (e.g., antibody-drug) conjugates.
\end{abstract}

Background: Antibody-drug conjugates play important roles in medical discovery for imaging and therapeutic intervention. The localization and stoichiometry of the conjugation can affect the orientation, selectivity, specificity, and strength of molecular interactions, influencing biochemical function.

Objective: To demonstrate the option to analyze the localization and stoichiometry of antibody-ligand conjugates by using essentially the same method at all levels including ligand infusion, peptide mapping, as well as reduced and intact protein analysis.

Materials and Methods: Capillary electrophoresis coupled with electrospray ionization mass spectrometry was used to analyze the antibody-ligand conjugates.

Results: We identified three prevalent ligand conjugation sites with estimated stoichiometries of 73,14 , and $6 \%$ and an average ligand-antibody ratio of 1.37 , illustrating the capabilities of CE-ESI-MS for rapid and efficient characterization of antibody-drug conjugates.

Conclusion: The developed multilevel analytical method offers a comprehensive way to determine the localization and stoichiometry of antibody-drug conjugates for molecular medicinal applications. In addition, a significant advantage of the reported approach is the small, hydrophilic, unmodified peptides well separated from the neutrals, which is not common with other liquid phase separation methods such as LC.

Keywords: Antibody-drug conjugates, protein small molecule conjugates, CE-MS, stoichiometry, characterization, unmodified peptides.

\section{INTRODUCTION}

Protein-small molecule conjugates (PSMCs) have distinct biological specificity and selectivity that make them powerful diagnostic tools and therapeutic agents, antibody-drug conjugates (ADCs) as a specific example [1-3]. Through either in vivo or in vitro linkage of a protein to a small molecule imaging agent, binding ligand, or drug, a variety of different biomedical functionalities can be created. Some of those functionalities include outstanding cytotoxicity in parallel with high selectivity, favorable pharmacokinetic profile (systemic circulation, enhanced permeability and retention) and improved stability. Application of PSMCs

\footnotetext{
*Address correspondence to this author at the Research Institute of Biomolecular and Chemical Engineering University of Pannonia, Egyetem street. 10, Veszprem, 8200 Hungary;

E-mail: jarvas@lendulet.uni-pannon.hu
}

as imaging agents holds great potential for precision cancer treatment due to their noninvasive and quantitative monitoring feature [4]. Antibody-imaging agent conjugates are usually dubbed as immunopositron emission tomography (PET). Tavare et al. published an excellent work about the development of an effective immuno-PET method to monitor CD8dependent responses to immunotherapy [5], where engineered anti-CD8 was linked to ${ }^{89} \mathrm{Zr}$ radiolabel to address a challenging cancer immunotherapy problem. $A D C s$, on the other hand, combine the site-specificity of a certain monoclonal antibody (mAb) with potential anti-cancer effect of a cytotoxic payload [6]. ADCs represent a new class of biopharmaceuticals; five have already been FDA approved and a few of them are in late-phase clinical testing [7]. Furthermore, imaging and therapy functions can be utilized simultaneously. Adumeau et al. reported a pioneering study about the 
development of a new methodology that facilitated the dual labeling of an antibody with both a toxin and a radionuclide [8].

In order to adequately maintain the activity of both protein and small molecule, a hydrophilic chemical linker often connects the two molecules. The stoichiometry of the small molecule on the protein affects specificity, selectivity, activity, and toxicity. In the case of biotherapeutics, the stoichiometry of the conjugate, referred to as drug-to-antibody ratio (DAR), is typically measured using UV absorbance and intact MS techniques. Similarly, the localization of the small molecule on the protein is an important parameter that can affect the binding properties of the conjugate. From a chemical analysis point of view, PSMCs are heterogeneous mixtures of molecules, whose complexity poses numerous analytical challenges. Such challenges are the determination of the free drug level (DAR stability over time most often measured by hydrophobic interaction chromatography), investigation of the residual solvents (media of the conjugation reaction) and the determination of antibody aggregation, in which latter has a tendency to increase due to the modification of the surface of the antibody [9]. Because of the combination of the antibody-, linker, and small-molecule chemistry, the complexity of PSMCs is much higher than that of any of the individual constituents. On the other hand, degradation pathways of PSMCs are very similar to those generally observed for other antibody based biopharmaceuticals. Physical and chemical stability of PSMCs with special emphasis on the conjugation process has been comprehensively reviewed by Ross and Wolfe [6].

Unlike traditional characterization of either the protein or the small molecule components, unique challenges arise to appropriately understand the purity and stability of the conjugates, as well as the small molecule-linker stoichiometry and their localization on the protein. Common analytical characterization of these protein conjugates by mass spectrometry includes intact and reduced mass analysis and peptide sequencing with post-translational modification identification, considering the small molecule conjugate [10-13]. Traditional analyses of either purified or synthesized proteins and small molecules are routinely and adequately performed by infusion and LC-MS. However, the added complexity of linking a protein and a small molecule creates additional challenges for common LC-MS strategies. In intact mass measurements, infusion and protein-compatible LC conditions result in co-elution and co-electrospray of PSMCs, particularly with the same stoichiometry, but different localizations. Co-elution/co-electrospray (ESI) during intact MS analysis is also a concern due to possible ion suppression and, thus, inaccurate estimation of relative stoichiometric ratios [14]. At the peptide level, it is difficult to elute using common chromatographic resins and elution methods/profiles [9, 15]. Thus, front-end separation methods for MS analysis would aid in the accurate and comprehensive characterization of PSMCs.
Capillary electrophoresis (CE) is a liquid phase analytical technique, well-suited for protein analysis, particularly when coupled with mass spectrometry. Specifically, capillary zone electrophoresis (CZE) is a mode of CE, which allows for separation of proteins and peptides in free solution without a stationary phase, i.e., only based on their charge and hydrodynamic volume ratios. The sensitivity of the method has significantly increased with the advent of laser induced fluorescence (LIF) detection technique [16].Recently, the integration of CE and ESI into a single capillary format (CESI) has improved the robustness and ionization efficiency to facilitate indepth characterization of biomolecules [17-21]. Some benefits of antibody drug conjugate analysis by CESIMS have already been demonstrated such as the quantification of the drug loading values of three druglysozyme conjugates by CESI-MS [22]. In this paper, we developed a new CE-ESI-MS method for the intact and reduced analysis of localization and stoichiometry of model antibody-ligand conjugates.

\section{MATERIALS AND METHODS}

FITC-PEG-NHS and the Fab domain were generated as previously described [23]. FITC-PEGNHS was analyzed at $10 \mathrm{mM}$ in $10 \%$ acetic acid, $\mathrm{pH}$ 3.1. Fab and Fab-PEG-FITC conjugate peptide samples were prepared at $1 \mathrm{mg} / \mathrm{mL}$ using a 4-hour digestion protocol with RapiGest (Waters, Milford, MA), DTT, iodoacetamide, and trypsin (all from Sigma, St Louis, MO), then diluted to $250 \mathrm{mg} / \mathrm{mL}$ in $125 \mathrm{mM}$ ammonium acetate $(\mathrm{pH} 4.0)$ as previously described $[23,24]$. Intact Fab and Fab-PEG-FITC samples were prepared at $1 \mathrm{mg} / \mathrm{mL}$ in $50 \mathrm{mM}$ ammonium acetate $(\mathrm{pH}$ 4.0).

CESI experiments were carried out with a CESI 8000 system (Sciex, Framingham, MA) equipped with a temperature-controlled autosampler and a power supply with the ability to deliver up to $30 \mathrm{kV}$ separation voltage. A commercial bare fused-silica OptiMS capillary cartridge (Sciex) with a porous tip was used for the infusion and peptide mapping experiments. $\mathrm{N}$ (trimethoxysilylpropyl) polyethyleneimine (PEI, Sigma) was used to coat the capillary for intact protein separations as described in U.S. Patent 6923895. Solutions of $10 \%$ acetic acid (peptide mapping) and $3 \%$ acetic acid (reduced and intact analysis) were employed as both background electrolytes (BGE) and conductive liquids. Pressure injections were used for FITC-PEG-NHS infusion (100 psi, $180 \mathrm{sec}$, capillary fill), peptide mapping (5 psi, $60 \mathrm{sec}, 50 \mathrm{~nL})$, as well as intact and reduced level protein ( $5 \mathrm{psi}, 10 \mathrm{sec}, \sim 8.5 \mathrm{~nL}$ ) analyses. Sample stacking after pressure injection was performed by transient isotachophoresis (t-ITP) [24]. CESI infusions and separations were performed at 20 $\mathrm{kV}$, except for the intact analysis using a PEl-coated capillary, which employed $-20 \mathrm{kV}$.

A TripleTOF® 6600 system with a NanoSpray® III source and CESI adapter were used operating at $2 \mathrm{kV}$ ion spray voltage (Sciex). MS/MS of the infused FITCPEG-NHS molecules were performed from 100 to 1000 
$\mathrm{m} / \mathrm{z}$ with a collisional energy of $40 \mathrm{~V}$. Peptide mapping was performed with information dependent acquisition (IDA) run with $15 \mathrm{MS} / \mathrm{MS}$ cycles from 250 to $2000 \mathrm{~m} / \mathrm{z}$. Intact and reduced protein analysis was performed with MS scans from $400-4500 \mathrm{~m} / \mathrm{z}$. High resolution MS and MS/MS spectra were analyzed using ProteinPilot $^{\mathrm{TM}}$, PeakView ${ }^{\circledR}$, and BioPharmaView ${ }^{\mathrm{TM}}$ softwares (Sciex) and the Intact Mass analysis software from Protein Metrics (Cupertino, CA).

\section{RESULTS}

A fluorescein isothiocyanate-polyethylene glycol-Nhydroxysuccinimide (FITC-PEG-NHS) ligand was randomly conjugated to primary amines on a fragment antigen-binding (Fab) domain at either the protein $\mathrm{N}$ terminus or the $\varepsilon$-lysine residues (Fig. 1A). The goal of the work was to characterize the localization and stoichiometry of the random Fab-ligand conjugates for structure and activity comparisons with a similar sitespecific Fab-PEG-FITC [23]. Using CESI both as an infusion and separation device for mass spectrometric analysis, the neat FITC-PEG-NHS and Fab molecules, the trypsin-digested peptide conjugates, as well as reduced and intact protein conjugates were all characterized.

Prior to the analysis of the Fab-PEG-FITC conjugate, the FITC-PEG-NHS and Fab molecules were analyzed individually. Infusion of the FITC-PEGNHS molecule by CESI allowed for the identification of FITC-PEG-specific signature fragment ions (Fig. 1B) used for peptide mapping data analysis. Intact mass analysis of the Fab fragment was also performed to measure the unconjugated protein and deconvoluted mass spectra (Fig. 1C and D). Peptide mapping data was generated and the signature FITC-PEG fragment ions were then used to extract candidate peptide conjugate electrophoretic peaks. Peptide conjugates were identified by both high resolution and high mass accuracy MS scans and MS/MS scans (Fig. 2) also quantified with extracted ion electropherograms (XIEs) (Fig. 3). The three most prevalent peptide conjugates were found at the heavy chain $\mathrm{N}$-terminus, heavy chain lysine 217, and light chain lysine 141 with estimated relative abundances of 73,14 , and $6 \%$ (Table 1), respectively. For the highest abundance $\mathrm{N}$-terminal localization, a PEG-only conjugation was also detected $(1.3 \%)$ at a different migration time. Three additional lower abundance peptide conjugates were also found at $2.4,0.7$, and $0.5 \%$ estimated relative abundances.

Intact level analysis by CESI-MS facilitated the separation of the different stoichiometries of the FabPEG-FITC molecule (Fig. 4A). Due to the CZE separation of Fab-PEG-FITC stoichiometric species, unique MS spectra (Fig. 4B) and deconvoluted spectra
(A)

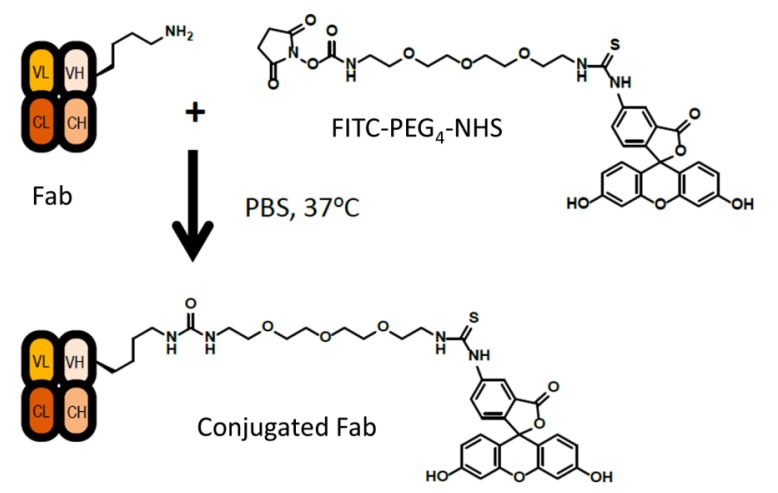

(C)

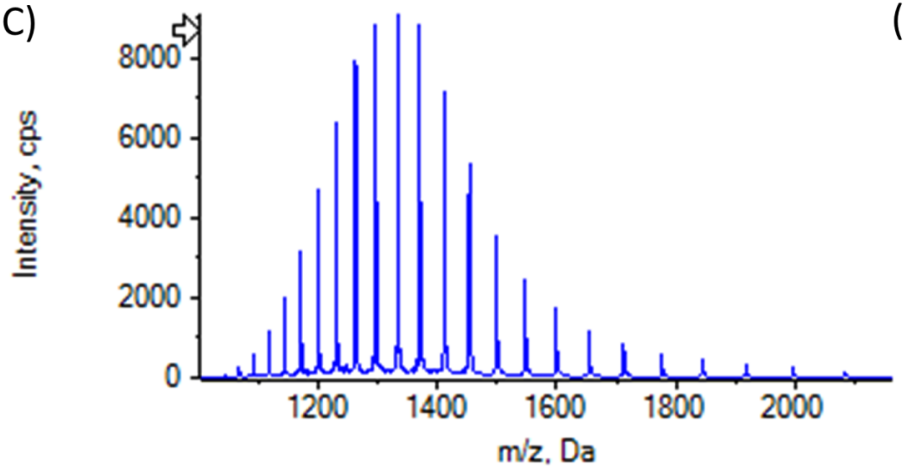

(B)

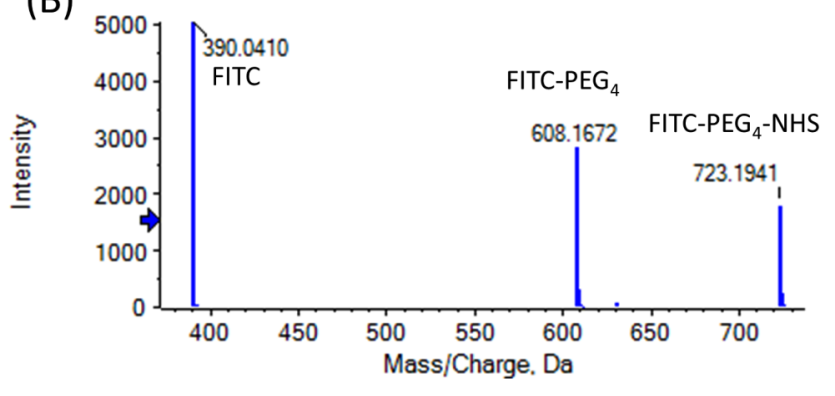

(D)

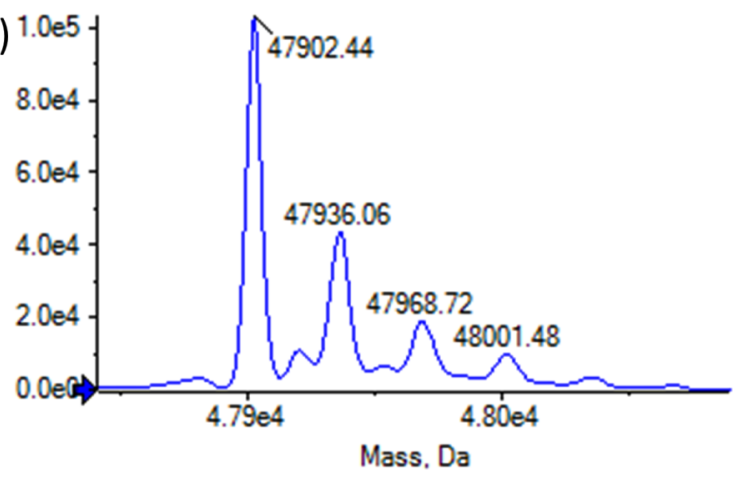

Fig. (1). A) General reaction scheme to generate random FITC-antibody conjugates with FITC-PEG4-NHS. (B) MS/MS spectra from an infusion of FITC-PEG4-NHS reagent. (C) Intact MS spectra of unconjugated Fab from a separate infusion. (D) Deconvoluted mass spectra of the unconjugated Fab. (A higher resolution / colour version of this figure is available in the electronic copy of the article). 

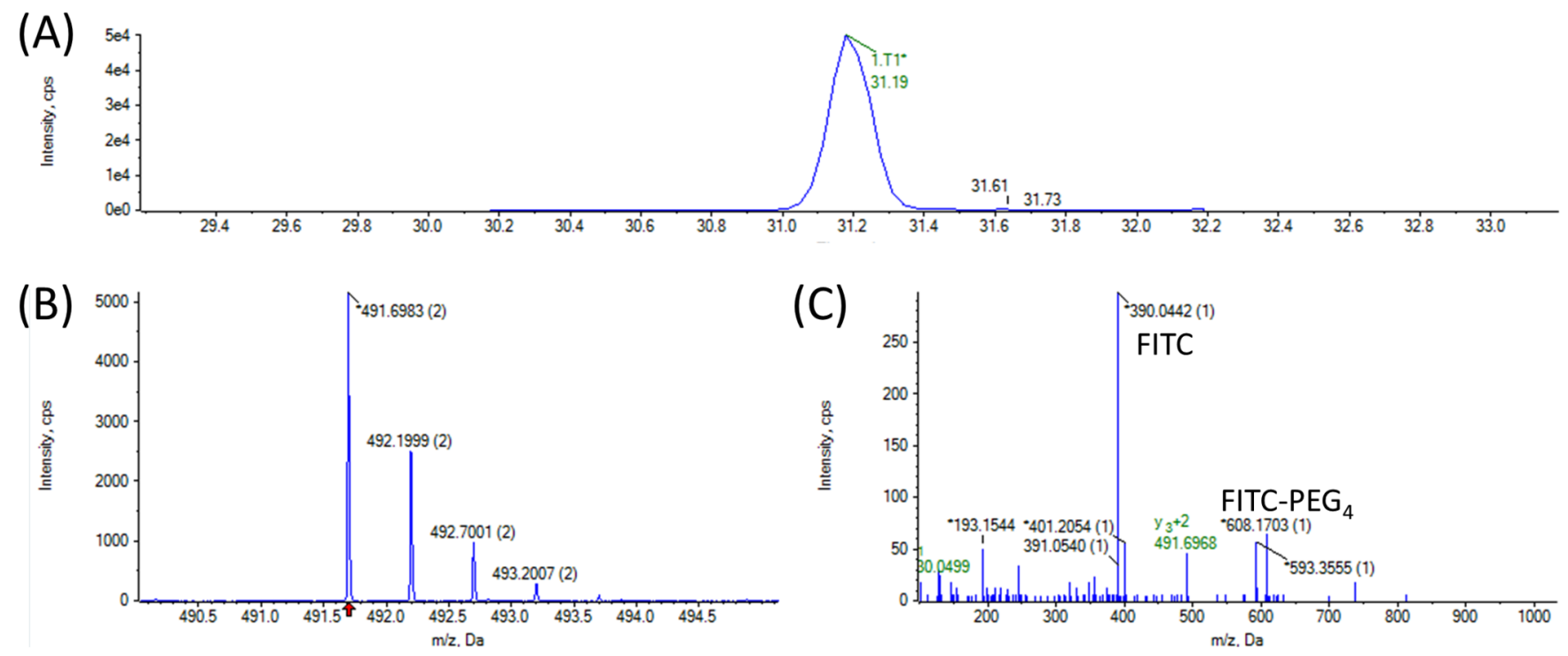

Fig. (2). Representative (A) extracted ion electropherogram, (B) intact MS spectra, and (C) MS/MS spectra with signature FITC and FITC-PEG 4 masses for the most abundant FITC-PEG - EVK peptide. (A higher resolution / colour version of this figure is available in the electronic copy of the article).
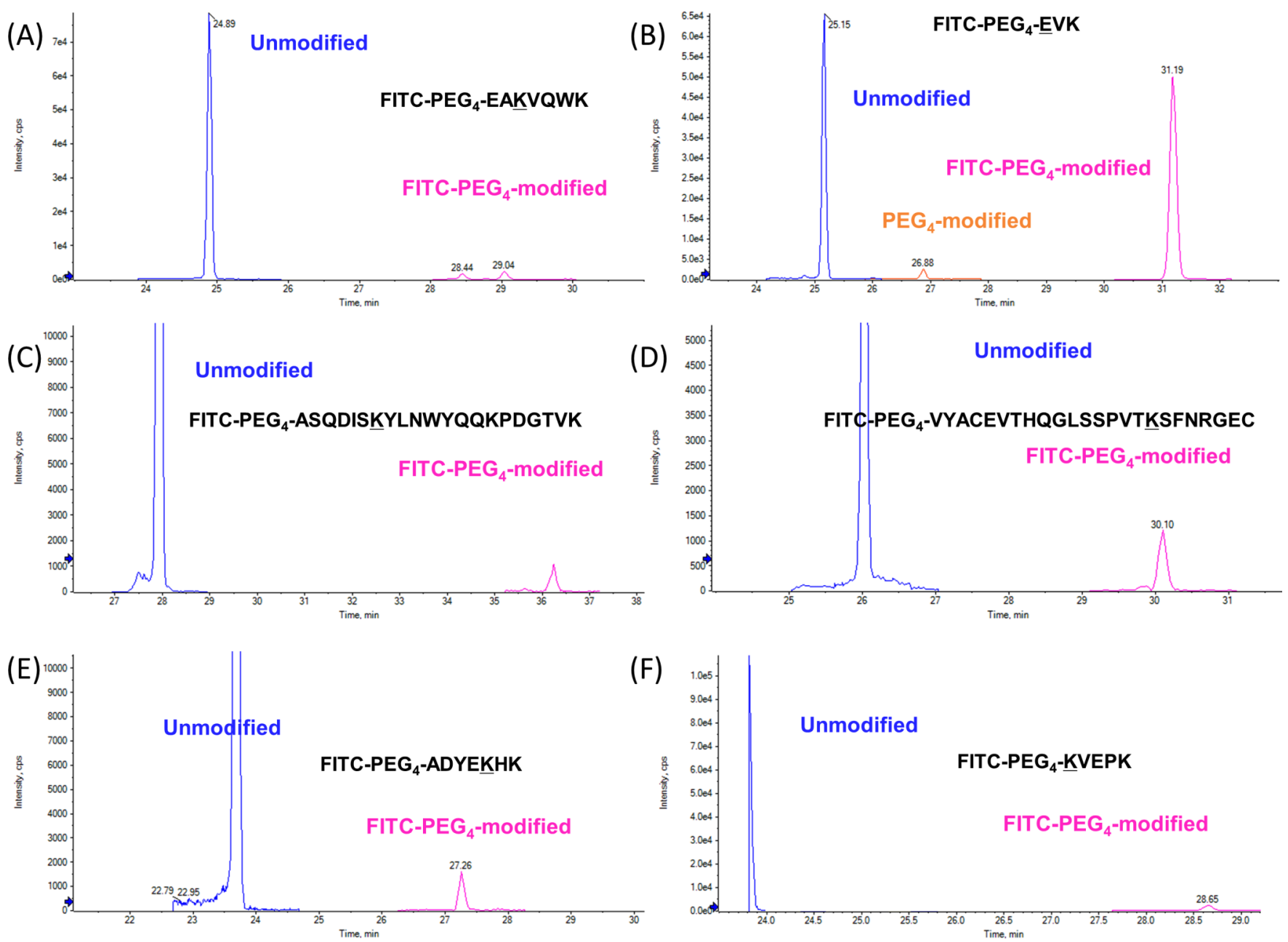

Fig. (3). Representative XIEs for modified and unmodified forms of FITC-PEG 4 -conjugated peptides used for relative abundance estimations in Table 1. (A higher resolution / colour version of this figure is available in the electronic copy of the article). 


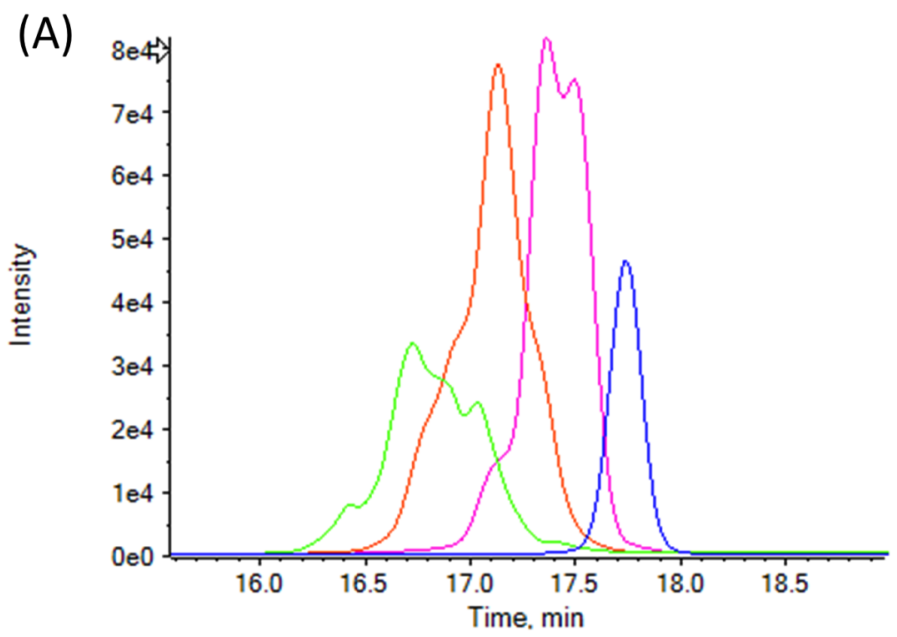

(C)
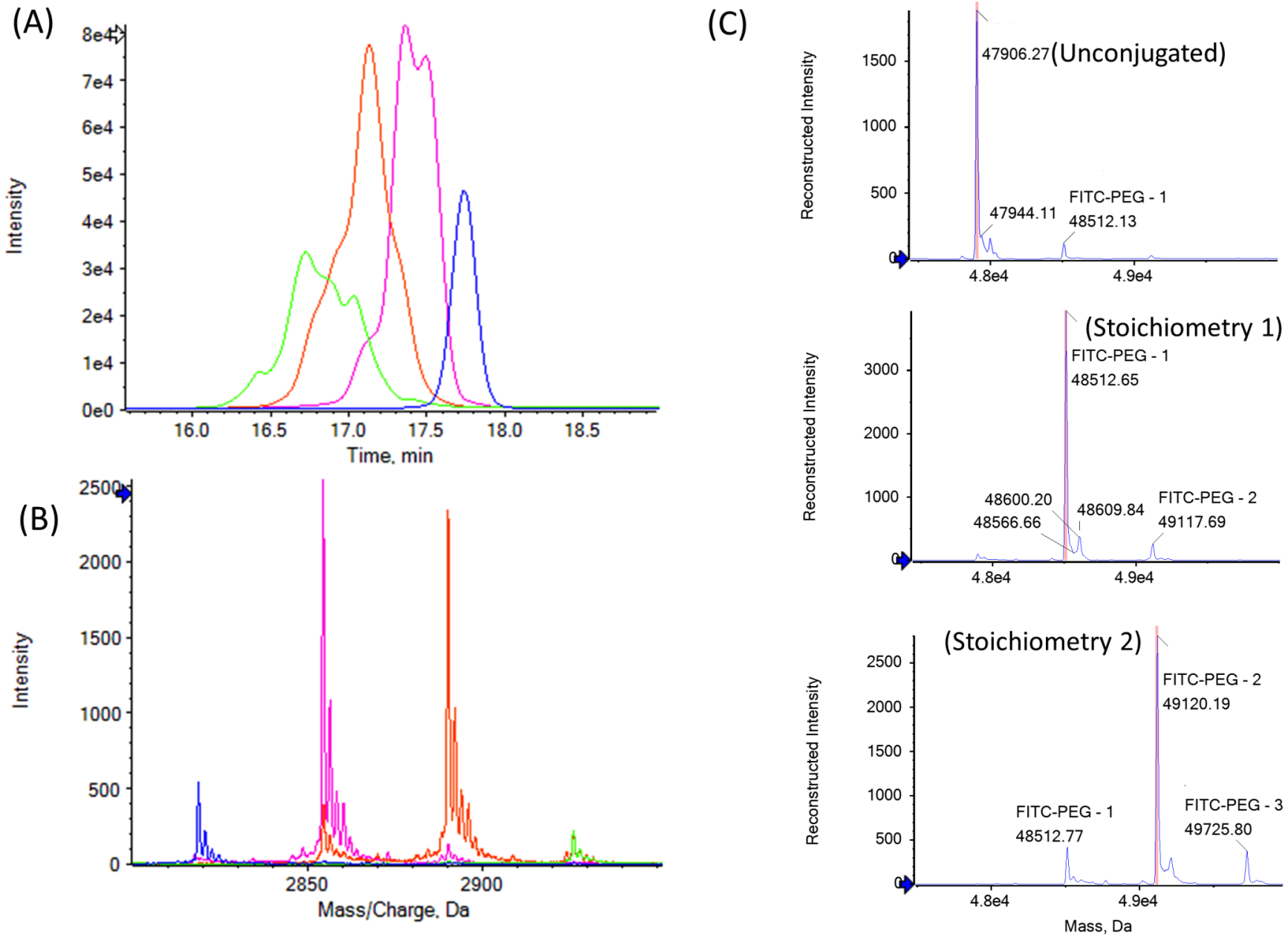

Fig. (4). (A) XIEs of intact Fab-PEG 4 -FITC masses from the most abundant charge state and isotope as shown in (B). Fab$\mathrm{PEG}_{4}$-FITC stoichiometries of zero (blue), one (pink), two (orange), and three (green) are represented in both (A) and (B). (C) Deconvoluted mass spectra of mass spectra shown in $(\mathrm{B})$. (A higher resolution / colour version of this figure is available in the electronic copy of the article).

(Fig. 4C) could also be generated for each species. From the intact MS data, the measured relative abundances $(12,38,36,13$, and $2 \%)$ for zero to four Fab-ligand stoichiometries from the intact analysis were estimated (Table 2), respectively, averaging a ligand-Fab ratio of 1.55 .

Reduced analysis by CESI-MS provided additional information about the Fab-PEG-FITC stoichiometries and structure. For instance, stoichiometry data from the reduced analysis (Table III) was consistent with both peptide mapping and intact analysis results. The majority of FITC-PEG conjugation was found on the heavy chain $(45.2 \%)$ with a stoichiometry of one, followed by the light chain $(24.5 \%)$ stoichiometry of one, and heavy chain (16.2\%) stoichiometry of two. Representative data for the most abundant heavy chain single conjugate and light chain without conjugation forms are shown in Fig. 5A. Additional analysis of the electrophoretic peaks and mass spectra indicated the presence of an unknown $14.9 \mathrm{kDa}$ polypeptide (Fig. 5B, Table 4).

\section{DISCUSSION}

The peptide mapping and intact measurements illustrated some important aspects of both non-specific conjugation of ligands and their analyses options, particularly with CESI-MS. Spatial resolution of PSMC at the intact and reduced levels by CESI-MS was complementary and supplementary to similar separations and results traditionally achieved at the peptide mapping level with LC-MS. While mass spectrometry was capable of differentiating the FITCPEG stoichiometries on the intact Fab fragment; based on mass alone $(\Delta 607 \mathrm{Da})$, the CZE-based separation facilitated the spatial resolution of the unique stoichiometric species prior to electrospray ionization. Thus, the generated unique intact and deconvoluted mass spectra (Fig. 4B) had a very little contribution in other stoichiometric species, indicative of very little comigration and co-electrospray. As mentioned earlier, this was important since co-electrospray can cause ion suppression of lower abundance or less efficiently ionized species. Ultimately this should provide a more 

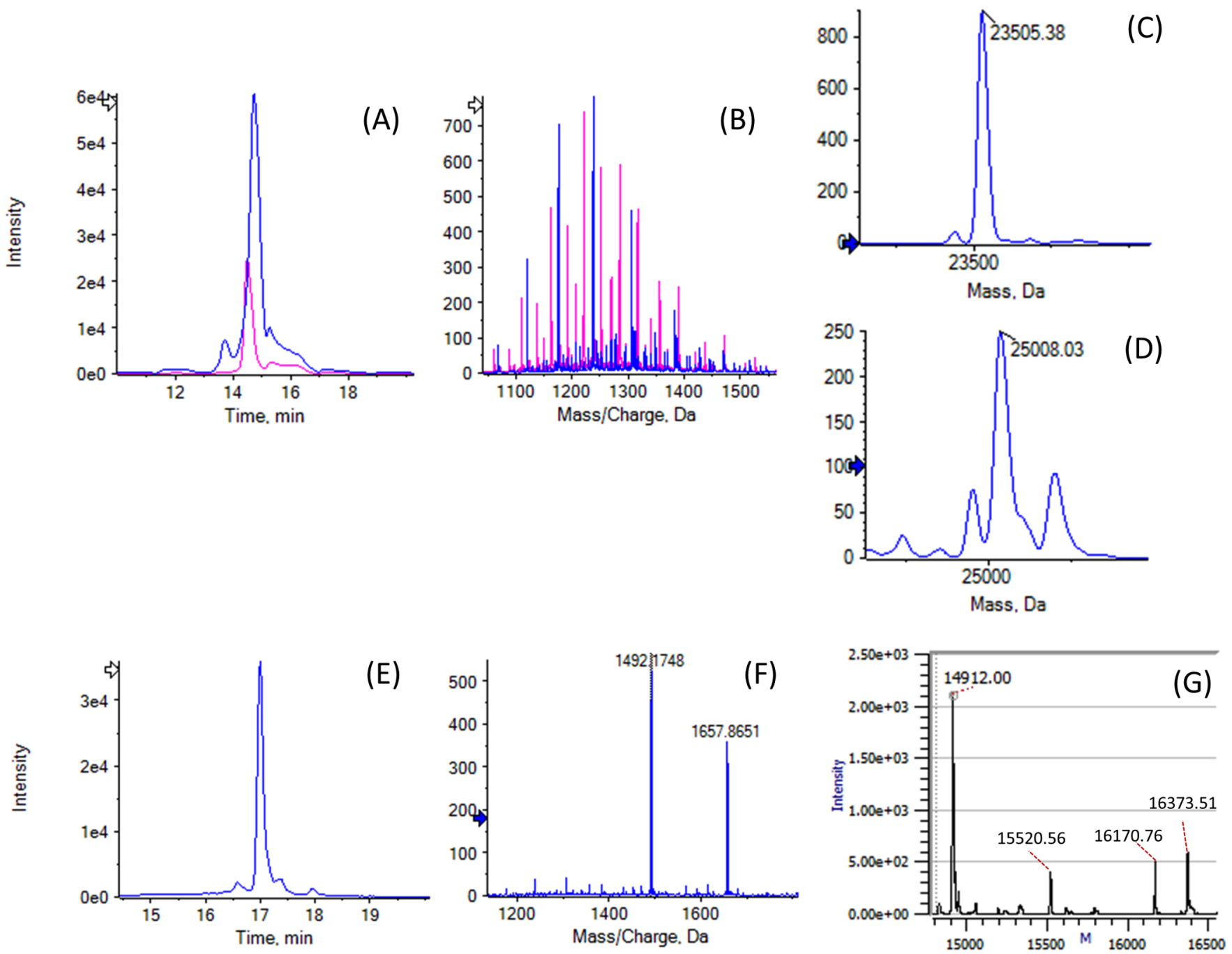

Fig. (5). Results from reduced analysis of Fab-PEG - FITC. (A) XIEs and (B) intact mass spectra of Fab light chain (blue) and Fab-PEG-FITC heavy chain (pink). Deconvoluted mass spectra of (C) Fab light chain and (D) Fab-PEG-FITC heavy chain. (E) $\mathrm{XIE},(\mathrm{F})$ intact mass spectra, and $(\mathrm{G})$ deconvoluted mass spectra of unexpected polypeptides. (A higher resolution / colour version of this figure is available in the electronic copy of the article).

accurate estimation of stoichiometry and DAR information of all fully intact species.

In the case of Fab-PEG-FITC conjugates, the CZEbased separation mechanism was primarily based on the change in the isoelectric point or charge of the protein in the acidic background electrolyte (BGE). For each conjugation of FITC-PEG to a primary amine on the Fab, a positive charge was removed from the FabPEG-FITC conjugate. This occurred for each additional FITC-PEG molecule added. Additionally, FITC itself has a carboxylic acid group, so it can also represent an additional negative charge on the Fab-PEG-FITC molecules under certain $\mathrm{pH}$ conditions. While this was most important to the intact protein analysis, the same separation mechanism applied to the reduced and peptide mapping analyses performed. In the case of peptide mapping data, the mobility shifts caused by the addition of a FITC-PEG molecule to a peptide were much greater (Fig. 3) since the charge to hydrodynamic volume ratio was also much greater for a peptide over an intact protein. For the peptides, the mobility shift was anywhere from four to eight minutes. The CZE separation was also capable of separating the PEGonly modified EAK peptide (Fig. 3B). Separation and identification of this peptide were particularly notable since (1) traditional peptide mapping by LC-MS would not retain or separate these species since they are very small and hydrophilic and (2) it could not be attributed to any ESI- or MS-based loss of FITC due to the large spatial resolution between the different EAK peptide forms. Thus, the PEG-only modified peptide would be indicative of loss of FITC from either the FITC-PEG$\mathrm{NHS}$ reagent before or during the labeling reaction or loss of the FITC after the conjugation step. In the case of a therapeutic ADC, this would represent the loss of the drug payload.

The CZE-based separation mechanism of the different species in solution was beneficial prior to ESI and MS detection of the Fab-PEG-FITC species for additional reasons. For example, the Fab-PEG-FITC stoichiometry was only detected at $2.2 \%$ relative abundance. It is unlikely that this low abundant form 
would have been detected accurately if coelectrosprayed with more abundant forms using either infusion or a co-eluting LC-MS strategy. Additionally, closer inspection of the intact Fab-PEG-FITC electrophoretic peaks (Fig. 4A) indicated that the peaks both broadened and split at higher stoichiometric ratios. In order to explain this phenomenon, the random nature of the conjugations must be considered. Since the conjugation to lysine residues is random and varies in abundance (Table 1), it would be expected that different combinations and efficiencies of FITC-PEG conjugations would create many different intact forms. For a Fab-PEG-FITC stoichiometry of one, there appear to be three partially unresolved peaks. This was consistent with three conjugation sites detected by peptide mapping at $\sim 10 \%$ and greater relative abundance. Similarly, considering the eight detected conjugations peptides, for a Fab-PEG-FITC stoichiometry of two, there are $2^{8}$ or 256 different possible intact forms. However, since only two peptides were detected at greater than $25 \%$ abundance (Table 1), it was likely that there could be $2^{2}$ (i.e., four) abundant intact Fab-PEG-FITC forms. Indeed, upon inspection of the electrophoretic peak profiles in Fig. 4A, there could be at least three partially unresolved intact forms. Finally, for a Fab-PEG-FITC stoichiometry of three, there are likely $3^{2}$ (i.e., 9 ) possible conjugation combinations again from the two conjugated peptides detected at about $25 \%$ relative abundance. Inspection of the electrophoretic peak associated with a stoichiometry of three indicated the partial resolution of $5-6$ peaks, also consistent with the expected number of combinations. This interpretation of the intact forms was also supported by the reduced level analysis. The extracted electrophoretic peak for the Fab-PET-FITC heavy chain stoichiometry of one is shown in Fig. 4A. As expected from the peptide mapping and intact analysis relative abundance measurements (Table $1 \&$ 2 ), there is one abundant form with at least two lower abundant forms present just for the HC Fab-PEG-FITC stoichiometry of one. Relative abundance measure- ments for all the different reduced Fab-PEG-FITC forms (Table 3) further supported the relative abundance and stoichiometry measurements made using peptide mapping and the intact analysis. Therefore, this data collectively shows that CESI-MS is well-suited for the peptide mapping, reduced, and intact level analysis of conjugated proteins, particularly when relative quantitation is of high importance.

Another notable advantage of using CESI-MS over traditional LC-MS methods is that very similar separation conditions can be used for small peptides (< $1 \mathrm{kDa})$ up to intact mAbs $(\sim 150 \mathrm{kDa})$. This capability is illustrated herein by the usage of BGEs only differing in acetic acid concentration ( $3 \%$ versus 10\%). For this reason, in either the peptide mapping, reduced or intact level analysis was possible to detect polypeptides of various sizes in the same analysis. Expected results of the reduced analysis of the Fab-PEG-FITC analysis are shown in Fig. 5A-D where the heavy and light chains are both present and their masses add up to the intact Fab-PEG-FITC mass. However, notably an abundant unknown $14.9 \mathrm{kDa}$ polypeptide was detected only in the reduced level analysis (Fig. 5E-G). Additional lower abundance polypeptides of $15.5,16.2$, and $16.4 \mathrm{kDa}$ were also detected at the same electrophoretic migration time (Fig. 5G). The lack of detection in the intact level implies the unknown polypeptide arose from a disulfide-linked form of the Fab fragment. The presence of this additional polypeptide may be best explained by the peptide mapping data. Two of the eight Fab-PEG-FITC peptides (Table 1, peptides MNSLQTDDTAIYYCAKHYYYGGSYAMDYWGQGTSV TVSSASTKGPSVFPLAPSSK $\underline{K}$ and_HYYYGGSYAM DYWGQGTSVTVSSASTE) were identified with Cterminal lysine conjugation with FITC-PEG. This would not be expected on a full-length heavy chain since trypsin is unable to cleave modified lysine residues [25, 26]. Thus, a probable explanation for the presence of these peptides is unexpected C-terminal lysine processing prior to the conjugation reaction. Both lysine-conjugate residues are near the middle of the

Table 1. List of identified FITC-PEG-peptides and PEG-peptides from peptide mapping.

\begin{tabular}{|c|c|c|c|}
\hline Chain & Modified amino acid and sequence & $\begin{array}{c}\text { FITC-PEG relative } \\
\text { abundance }^{*}\end{array}$ & $\begin{array}{c}\text { PEG relative } \\
\text { abundance* }^{*}\end{array}$ \\
\hline \hline Heavy & E1, EVK & $73.3 \%$ & $1.3 \%$ \\
\hline Heavy & MNSLQTDDTAIYYCAKHYYYGGSYAMDYWGQGTSVTVSSASTKG & $27.8 \%$ & $27.8 \%{ }^{*}$ \\
\hline Seavy & K217, KVEPK & $14.4 \%$ & Not detected \\
\hline Light & K141, EAKVQWK & $6.1 \%$ & Not detected \\
\hline Heavy & K124, HYYYGGSYAMDYWGQGTSVTVSSASTKK & $3.3 \%$ & Not detected \\
\hline Light & K31, ASQDISKKYLNWYQQKPDGTV & $2.4 \%$ & Not detected \\
\hline Light & K188, ADYEKHK & $0.7 \%$ & Not detected \\
\hline Light & K207, VYACEVTHQGLSSPVTKSFNRGE & $0.5 \%$ & Not detected \\
\hline
\end{tabular}

*Relative abundances were calculated by dividing summed peak areas of modified peptides by summed peak areas of all associated peptide sequences at different charge states. PEG and FITC-PEG relative abundance are the same since both were detected on the same peptide. 
heavy chain (K124 and K136 out of 228 residues). Notably, the theoretical masses of the $\mathrm{N}$-terminal heavy chain polypeptides truncated at $\mathrm{K} 124$ and $\mathrm{K} 136$ are 13.6 and $14.8 \mathrm{kDa}$, respectively. The $14.8 \mathrm{kDa}$ theoretical mass (Table 4) is strikingly similar to the $14.9 \mathrm{kDa}$ measured mass (Fig. 5G and Table 4). Additionally, other lower abundant masses of 15.5 , 16.2 , and $16.4 \mathrm{kDa}$ correlate well with anticipated masses of the conjugated forms of the hypothesized 14.9 kDa polypeptide (Fig. 5G and Table 4). Further, MS/MS characterization of the $14.9 \mathrm{kDa}$ polypeptide and lower abundance modified forms would be needed to confirm this hypothesis.

Table 2. Estimation of Fab-ligand stoichiometries from intact analysis.

\begin{tabular}{|c|c|c|}
\hline No. of FITC-PEG per Fab & Peak Areas $^{*}$ & \% Peak Area \\
\hline \hline 0 & $6.17 \times 10^{3}$ & $11.8 \%$ \\
\hline 1 & $1.98 \times 10^{4}$ & $37.9 \%$ \\
\hline 2 & $1.85 \times 10^{4}$ & $35.5 \%$ \\
\hline 3 & $6.55 \times 10^{3}$ & $12.6 \%$ \\
\hline 4 & $1.16 \times 10^{3}$ & $2.2 \%$ \\
\hline
\end{tabular}

*Peak areas were calculated by summing the signal for all associated charge states for each Fab-PEG-FITC stoichiometry using the Analyst software (Sciex).

Table 3. Estimation of Fab-ligand stoichiometries from reduced analysis.

\begin{tabular}{|c|c|c|}
\hline $\begin{array}{c}\text { No. of FITC-PEG per } \\
\text { Fab chain }\end{array}$ & Peak Areas* & \% Peak Area \\
\hline \hline Heavy (0) & $1.23 \times 10^{3}$ & $38.6 \%$ \\
\hline Heavy (1) & $1.44 \times 10^{3}$ & $45.2 \%$ \\
\hline Heavy (2) & $5.15 \times 10^{2}$ & $16.2 \%$ \\
\hline Light (0) & $9.75 \times 10^{3}$ & $75.5 \%$ \\
\hline Light (1) & $3.17 \times 10^{3}$ & $24.5 \%$ \\
\hline
\end{tabular}

${ }^{*}$ Peak areas were calculated by summing the signal for all associated charge states for each Fab-PEG-FITC stoichiometry.

Table 4. Comparison of measured and theoretical masses for truncated heavy chain species.

\begin{tabular}{|c|c|c|}
\hline $\begin{array}{c}\text { Heavy chain C E1 - K136 } \\
\text { modifications }\end{array}$ & Theoretical mass & $\begin{array}{c}\text { Measured } \\
\text { mass }\end{array}$ \\
\hline \hline None & 14819.75 & 14912.00 \\
\hline FITC-PEG & 15426.91 & 15520.56 \\
\hline 2 FITC-PEG & 16034.07 & 16170.76 \\
\hline 2 FITC-PEG + PEG & 16252.20 & 16373.51 \\
\hline
\end{tabular}

These types of in-depth considerations with higher separation resolving power are important not only for the experiments described herein, but also for the analysis of other lysine-conjugated or non-specifically conjugated ligands on other protein and antibody conjugates used as diagnostics and therapeutics. Collectively, these results illustrate the capabilities of
CESI-MS for the characterization of antibody-ligand conjugates with a multi-functional workflow at the small molecule, peptide, reduced, and intact protein levels.

\section{LIST OF ABBREVIATIONS}

$\mathrm{CESI}=$ capillary electrophoresis electrospray ionization

$\mathrm{ADC}=$ antibody-drug conjugate

PSMC $=$ protein-small molecule conjugate

$\mathrm{PET} \quad=$ positron emission tomography

$\mathrm{mAb}=$ monoclonal antibody

DAR = drug-to-antibody ratio

CZE $=$ capillary zone electrophoresis

PEG = polyethylene glycol

FITC = fluorescein isothiocyanate

$\mathrm{Fab}=$ fragment antigen-binding

$\mathrm{XIE}=$ extracted ion electropherogram

\section{ETHICS APPROVAL AND CONSENT TO PARTICIPATE}

Not applicable.

\section{HUMAN AND ANIMAL RIGHTS}

No Animals/Humans were used for studies that are the basis of this research.

\section{CONSENT FOR PUBLICATION}

Not applicable.

\section{AVAILABILITY OF DATA AND MATERIALS}

Not applicable.

\section{FUNDING}

The authors gratefully acknowledge the grants of the National Research, Development and Innovation Office (NN127062, 2018-2.1.17-TÉT-KR-2018-00010). This work was also sponsored by the BIONANO_GINOP-2.3.2-15-2016-00017 project and by the János Bolyai Research Scholarship as well as the ÚNKP-19-4 New National Excellence Program of The Ministry for Innovation and Technology. This is contribution \# 162 from the Horváth Csaba Memorial Laboratory of Bioseparation Sciences.

\section{CONFLICT OF INTEREST}

The authors declare no conflict of interest, financial or otherwise.

\section{ACKNOWLEDGEMENTS}

The authors acknowledge Chan Hyuk Kim of Calibr at the time, and now KAIST, for providing Fab-PEGFITC reagents and conjugated samples; and St John 
Skilton and Eric Carlson of Protein Metrics Inc for access and assistance with their Intact Mass software and Eric Johansen at the time of SCIEX, now Amunix, for MS advise and technical assistance.

\section{REFERENCES}

[1] Casi G, Neri D. Antibody-Drug Conjugates and Small Molecule-Drug Conjugates: Opportunities and Challenges for the Development of Selective Anticancer Cytotoxic Agents. J Med Chem 2015; 58(22): 8751-61.

http://dx.doi.org/10.1021/acs.jmedchem.5b00457 PMID: 26079148

[2] Birrer MJ, Moore KN, Betella I, Bates RC. Antibody-Drug Conjugate-Based Therapeutics: State of the Science. J Natl Cancer Inst 2019; 111(6): 538-49.

http://dx.doi.org/10.1093/jnci/djz035 PMID: 30859213

[3] Beck A, Goetsch L, Dumontet C, Corvaïa N. Strategies and challenges for the next generation of antibody-drug conjugates. Nat Rev Drug Discov 2017; 16(5): 315-37. http://dx.doi.org/10.1038/nrd.2016.268 PMID: 28303026

[4] Carmon KS, Azhdarinia A. Application of Immuno-PET in Antibody-Drug Conjugate Development, Mol Imaging 2018; 17: 1536012118801223-1536012118801223..

[5] Tavaré R, Escuin-Ordinas $\mathrm{H}$, Mok $\mathrm{S}$, et al. An Effective Immuno-PET Imaging Method to Monitor CD8-Dependent Responses to Immunotherapy. Cancer Res 2016; 76(1): 7382.

http://dx.doi.org/10.1158/0008-5472.CAN-15-1707 PMID: 26573799

[6] Ross PL, Wolfe JL. Physical and Chemical Stability of Antibody Drug Conjugates: Current Status. J Pharm Sci 2016; 105(2): 391-7.

http://dx.doi.org/10.1016/j.xphs.2015.11.037 PMID: 26869406

[7] Thomas A, Teicher BA, Hassan R. Antibody-drug conjugates for cancer therapy. Lancet Oncol 2016; 17(6): e254-62. http://dx.doi.org/10.1016/S1470-2045(16)30030-4 PMID: 27299281

[8] Adumeau P, Vivier D, Sharma SK, et al. Site-Specifically Labeled Antibody-Drug Conjugate for Simultaneous Therapy and ImmunoPET. Mol Pharm 2018; 15(3): 892-8.

http://dx.doi.org/10.1021/acs.molpharmaceut.7b00802 PMID: 29356543

[9] Wagh A, Song H, Zeng M, Tao L, Das TK. Challenges and new frontiers in analytical characterization of antibody-drug conjugates. MAbs 2018; 10(2): 222-43.

http://dx.doi.org/10.1080/19420862.2017.1412025 PMID: 29293399

[10] Schnaible V, Przybylski M. Identification of fluorescein-5'isothiocyanate-modification sites in proteins by electrosprayionization mass spectrometry. Bioconjug Chem 1999; 10(5): 861-6.

http://dx.doi.org/10.1021/bc990039x PMID: 10502354

[11] Manikwar P, Zimmerman T, Blanco FJ, Williams TD, Siahaan TJ. Rapid identification of fluorochrome modification sites in proteins by LC ESI-Q-TOF mass spectrometry. Bioconjug Chem 2011; 22(7): 1330-6.

http://dx.doi.org/10.1021/bc100560c PMID: 21612301

[12] Nguyen JM, Smith J, Rzewuski S, Legido-Quigley C, Lauber MA. High sensitivity LC-MS profiling of antibody-drug conjugates with difluoroacetic acid ion pairing. MAbs 2019; 11(8): 1358-66.

http://dx.doi.org/10.1080/19420862.2019.1658492 PMID: 31500514

[13] Jayathirtha M, Darie CC. Investigation of Antibody-Drug Conjugates by Mass Spectrometry. Adv Exp Med Biol 2019; 1140: 251-63. http://dx.doi.org/10.1007/978-3-030-15950-4_14 PMID: 31347052

[14] Donnelly DP, Rawlins CM, DeHart CJ, et al. Best practices and benchmarks for intact protein analysis for top-down mass spectrometry. Nat Methods 2019; 16(7): 587-94.

http://dx.doi.org/10.1038/s41592-019-0457-0

PMID: 31249407

[15] Li Y, Gu C, Gruenhagen J, Yehl P, Chetwyn NP, Medley CD An enzymatic deconjugation method for the analysis of small molecule active drugs on antibody-drug conjugates. MAbs 2016; 8(4): 698-705.

http://dx.doi.org/10.1080/19420862.2016.1151590 PMID 26891281

[16] Szöke E, Tábi T. Analysis of biological samples by capillary electrophoresis with laser induced fluorescence detection. $J$ Pharm Biomed Anal 2010; 53(5): 1180-92. http://dx.doi.org/10.1016/j.jpba.2010.07.045 PMID: 20719451

[17] Gulersonmez MC, Lock S, Hankemeier T, Ramautar R. Sheathless capillary electrophoresis-mass spectrometry for anionic metabolic profiling. Electrophoresis 2016; 37(7-8): 1007-14.

http://dx.doi.org/10.1002/elps.201500435 PMID: 26593113

[18] Jarvas G, Fonslow B, Yates JR III, Foret F, Guttman A. Characterization of a Porous Nano-electrospray Capillary Emitter at Ultra-low Flow Rates. J Chromatogr Sci 2017; 55(1): 47-51.

http://dx.doi.org/10.1093/chromsci/bmw148 PMID: 27993863

[19] Lageveen-Kammeijer GSM, de Haan N, Mohaupt P, et al. Highly sensitive CE-ESI-MS analysis of $\mathrm{N}$-glycans from complex biological samples. Nat Commun 2019; 10(1): 2137. http://dx.doi.org/10.1038/s41467-019-09910-7

PMID: 31086181

[20] Snyder CM, Zhou X, Karty JA, Fonslow BR, Novotny MV, Jacobson SC. Capillary electrophoresis-mass spectrometry for direct structural identification of serum N-glycans. J Chromatogr A 2017; 1523: 127-39.

http://dx.doi.org/10.1016/j.chroma.2017.09.009 PMID: 28989033

[21] Faserl K, Sarg B, Lindner HH. Application of CE-MS for the analysis of histones and histone modifications Methods (San Diego, Calif) 2020.

[22] Haselberg R, Harmsen S, Dolman MEM, de Jong GJ, Kok RJ, Somsen GW. Characterization of drug-lysozyme conjugates by sheathless capillary electrophoresis-time-offlight mass spectrometry. Anal Chim Acta 2011; 698(1-2): 7783.

http://dx.doi.org/10.1016/j.aca.2011.04.050 PMID: 21645662

[23] Ma JS, Kim JY, Kazane SA, et al. Versatile strategy for controlling the specificity and activity of engineered $T$ cells. Proc Natl Acad Sci USA 2016; 113(4): E450-8. http://dx.doi.org/10.1073/pnas.1524193113 PMID: 26759368

[24] Lew C, Gallegos-Perez JL, Fonslow B, Lies M, Guttman A. Rapid level-3 characterization of therapeutic antibodies by capillary electrophoresis electrospray ionization mass spectrometry. J Chromatogr Sci 2015; 53(3): 443-9.

http://dx.doi.org/10.1093/chromsci/bmu229 PMID: 25681206

[25] Xu G, Jaffrey SR. Proteomic identification of protein ubiquitination events. Biotechnol Genet Eng Rev 2013; 29: 73-109.

http://dx.doi.org/10.1080/02648725.2013.801232 PMID: 24568254

[26] Peng J, Schwartz D, Elias JE, et al. A proteomics approach to understanding protein ubiquitination. Nat Biotechnol 2003 21(8): 921-6.

http://dx.doi.org/10.1038/nbt849 PMID: 12872131 